\title{
Muscle Spindle-Derived Neurotrophin 3 Regulates Synaptic Connectivity between Muscle Sensory and Motor Neurons
}

\author{
Hsiao-Huei Chen, ${ }^{1}$ Warren G. Tourtellotte, ${ }^{2}$ and Eric Frank ${ }^{1}$ \\ ${ }^{1}$ Department of Neurobiology, University of Pittsburgh, Pittsburgh, Pennsylvania 15261, and 2Department of Pathology \\ (Neuropathology), Neurology and Neuroscience Institute, Northwestern University School of Medicine, Chicago, Illinois \\ 60611
}

la afferents induce the formation of muscle spindles prenatally and maintain them postnatally. To address whether spindles, in turn, regulate the function of la afferents, we examined Egr3null mutant mice (Egr3 ${ }^{-1-}$ ), in which muscle spindles degenerate progressively after birth. Egr $3^{-1-}$ mice develop gait ataxia, scoliosis, resting tremors, and ptosis, suggesting a defect in proprioception. Despite the normal morphological appearance of peripheral and central sensory projections, we observed a profound functional deficit in the strength of sensory-motor

Muscle spindles are specialized sense organs that respond to muscle stretch and provide information about axial and limb position (proprioception) to the CNS. They are innervated by sensory (group Ia and II) and motor $(\gamma)$ axons. Sensory innervation during prenatal stages provides trophic signals that induce the differentiation of primary myotube precursors and encapsulation of muscle fibers into a functional muscle spindle (Kucera and Walro, 1987, 1988; Zelena, 1994). The postnatal maintenance of spindles also requires sensory innervation; muscle deafferentation during the first postnatal week results in the degeneration of spindles (Kucera and Walro, 1987, 1988).

Conversely, proprioceptive sensory afferents, including those supplying muscle spindles, require their peripheral muscle targets for survival during fetal development (Carr and Simpson, 1978; Hamburger et al., 1981). Removal of limb buds at embryonic stages eliminates most muscle sensory neurons (Oakley et al., 1997), suggesting that trophic factors derived from muscles are critical for their survival. One possible factor is neurotrophin 3 (NT3). Proprioceptive afferents express the tyrosine kinase C (trkC) receptor, and fetal muscles express NT3, the major trkC ligand. The null mutation of NT3 results in significant cell death of these trkC $^{+}$sensory neurons (Ernfors et al., 1994; Farinas et al., 1994; Tessarollo et al., 1994; Tojo et al., 1995). As axons of trkC ${ }^{+}$proprioceptive neurons reach their peripheral targets, muscle-derived NT3 becomes important for neuron survival (Oakley et al., 1995, 1997; Wright et al., 1997). Injection of NT3 antibodies into peripheral tissues decreases the number of proprioceptive neurons (Oakley et al., 1995). Moreover, exogenous supplementation of NT3 can rescue muscle sensory neurons even

Received Oct. 1, 2001; revised Dec. 13, 2001; accepted Jan. 28, 2002.

This work was supported by National Institutes of Health grants to E.F. and W.G.T. H.-H.C. is supported by a National Research Service Award fellowship. We thank Alexandre F. R. Stewart and David R. Ladle for helpful discussions and Stan B. Convington for his excellent technical support.

Correspondence should be addressed to Eric Frank, Department of Neurobiology, University of Pittsburgh, Pittsburgh, PA 15261. E-mail: efrank@pitt.edu. Copyright (C) 2002 Society for Neuroscience $0270-6474 / 02 / 223512-08 \$ 15.00 / 0$ connections in Egr3 ${ }^{-1-}$ mice. Muscle spindles in Egr3 ${ }^{-/-}$mice do not express NT3. Intramuscular injections of NT3 to Egr3 ${ }^{-/-}$ mice during the postnatal period restored sensory-motor connections. Thus, NT3 derived from muscle spindles regulates the synaptic connectivity between muscle sensory and motor neurons.

Key words: muscle spindle; NT3; Egr3; la afferent; proprioceptive neuron; motoneuron; trophic factor in the absence of their peripheral targets. The number of muscle sensory neurons that survive depends on the levels of NT3 in the periphery (Oakley et al., 1997). Even in normal embryos, elevated NT3 increases the number of muscle afferents that survive (Oakley et al., 1997; Wright et al., 1997). Thus, muscle-derived NT3 is important for regulating the number of muscle sensory neurons during embryonic development.

The role of peripheral NT3 on the postnatal function of intact muscle spindle afferents has never been examined directly in vivo. In adult rats and cats, however, transection of peripheral muscle nerves leads to a reduction in the strength of synaptic inputs from Ia afferents to motoneurons, suggesting that a peripherally derived factor might normally maintain these synapses (Goldring et al., 1980). Moreover, exogenous NT3 supplied to the central nerve stump can block this reduction, maintaining central connectivity (Munson et al., 1997; Mendell et al., 1999). Whether NT3 is also required for maintenance of synaptic connectivity normally, in the absence of axotomy, is not known.

To examine the postnatal influence of muscle spindles on proprioceptive sensory afferents, we make use of a mutant mouse in which spindles regress after birth (Tourtellotte et al., 2001). The zinc-finger transcription factor Egr3 is normally expressed in developing intrafusal muscle fibers shortly after Ia afferents innervate muscle, but it is not expressed in sensory or motor neurons. In Egr3-null mutant mice $\left(\operatorname{Egr} 3^{-/-}\right)$, spindles are present at birth, but most disappear by adulthood. The mutant mice develop gait ataxia, scoliosis, resting tremors, and ptosis, suggesting a defect in proprioception. Here, we report a profound functional deficit in sensory-motor connections that develops postnatally in the spinal cord of Egr3 $3^{-1-}$ mice. Spindles in neonatal Egr3 ${ }^{-1-}$ mice do not express NT3, and synaptic connections can be restored by intramuscular injection of NT3 during the first two postnatal weeks. These experiments provide the first direct evidence for a postnatal trophic effect of muscle spindles on sensory-motor synaptic connectivity, an effect mediated largely by NT3 produced by intrafusal muscle fibers. 


\section{MATERIALS AND METHODS}

Electrophysiology. We examined the sensory input to motoneurons in an isolated spinal cord preparation (for details, see Mears and Frank, 1997; Arber et al., 2000). Synaptic potentials were recorded from motor axons in the ventral root [lumbosacral (L) segment 3 for quadriceps and obturator muscles and L5 for soleus and gastrocnemius muscles] with a tight-fitting suction electrode in response to electrical stimulation of various muscle nerves. In some cases, the soleus muscle or the rectus femoris (RF) head of the quadriceps was isolated with the spinal cord. To activate Ia afferents selectively, we applied 1-2 msec, $\sim 50 \mu \mathrm{m}$ stretches to the distal tendon of the RF or soleus muscle with a piezoelectric bimorph (Brown et al., 1967; Lichtman and Frank, 1984; Arber et al., 2000). Ib afferents were selectively stimulated by eliciting singletwitch muscle contractions (Jansen and Rudjord, 1964) via electrical stimulation of the L3 (for RF) or L5 (for soleus) ventral root (below threshold for $\gamma$ fiber activation). Synaptic potentials were stored digitally and measured from averages of $10-20$ traces at $0.5 \mathrm{~Hz}$ using custom software.

To measure the monosynaptic component of the evoked potentials, we scaled a superimposed model trace of proprioceptive input from the same muscle nerve in a normal mouse of similar age (Sah and Frank, 1984; Mears and Frank, 1997; Arber et al., 2000). The model trace was evoked by stimulating a muscle nerve at just suprathreshold strength; the resulting $(10-20 \mu \mathrm{V})$ synaptic potential therefore contained few, if any, polysynaptic components. To adjust for differences in the conduction velocities of Ia afferents in mutant mice, we measured the time of arrival of these impulses in the ventral horn using the terminal field potential (Watt et al., 1976; Munson and Sypert, 1979; Arber et al., 2000). This potential represents ephaptic coupling between Ia afferent arbors and motoneuronal dendrites and is visible in ventral root recordings (see Figs. $1 A, 8 D$ ). The latency of the terminal field potential was then used to adjust the position of the monosynaptic model along the time axis. In addition, we also recorded monosynaptic model traces in the NT3treated mutant mice; the timing of monosynaptic responses in these mice agreed with the adjustment derived from the delay in the field potential. When inputs were large enough to evoke orthodromic action potentials in motoneurons, as seen in the response to stimulation of the obturator nerve shown in Figure 1, the model trace was scaled to match the rising phase of the response, before the action potential occurred.

Retrograde labeling of central projections of sensory neurons. To examine the central projections of sensory neurons, we applied HRP to the L3 dorsal root at postnatal day 8 (P8). The ventral roots were cut before labeling to avoid retrograde labeling of motoneurons. The cord was processed conventionally, as described by Sah and Frank (1984).

NT3 administration. NT3 (a gift from Regeneron Pharmaceuticals, Inc., Tarrytown, NY) was injected (5 $\mathrm{g} / \mathrm{gm})$ in a volume of $1-4 \mu \mathrm{l}$ of PBS into the right proximal hindlimb of $\mathrm{Egr}^{-1-}$ mice using three different schedules: P1, P3, P5, P7; P5, P7, P9, P11; and P5, P6, P7.

In situ hybridization. $\mathrm{P} 0$ muscles were fixed with $4 \%$ paraformaldehyde and sectioned on a cryostat at $20 \mu \mathrm{m}$. Sections were hybridized with NT3 riboprobe labeled with digoxigenin (Roche, Indianapolis, IN) (Wright et al., 1997) and detected with alkaline phosphatase-conjugated antidigoxigenin antibody using a 5-bromo-4-chloro-3-indolyl phosphate/nitroblue tetrazolium kit (Vector Laboratories, Burlingame, CA).

Immunohistochemistry. P0 or P12 muscles were prefixed in cold acetone for 5-10 min, rinsed briefly with PBS, and cryoprotected by overnight immersion in $30 \%$ sucrose in PBS. Cryostat sections of $20 \mu \mathrm{m}$ thickness were post-fixed with $4 \%$ paraformaldehyde for $5 \mathrm{~min}$, rinsed in PBS, preincubated with blocking buffer (20\% goat serum in PBS) for 1 $\mathrm{hr}$, and incubated with anti-NT3 (1:1000; Chemicon, Temecula, CA) or anti-calbindin (1:1000; SWant, Bellinoza, Switzerland) rabbit antibody overnight at $4^{\circ} \mathrm{C}$. The biotinylated secondary antibodies were detected by avidin-conjugated alkaline phosphatase using diaminobenzidine (DAB)/ $\mathrm{H}_{2} \mathrm{O}_{2}$ (avidin-biotin complex and DAB kits from Vector Laboratories).

Spindle counts. Spindles stained with calbindin antibody were counted in every fifth $20 \mu \mathrm{m}$ cryostat section throughout the length of the quadriceps muscle. Spindles detected at the same location in adjacent fifth sections were counted only once, so as to eliminate duplicate counts.

\section{RESULTS}

Monosynaptic connections between la afferents and motoneurons are dysfunctional in Egr3 ${ }^{-1-}$ mice

The ataxic gait of Egr3-null mutant mice suggested that these mice might have a defect in proprioception. Muscle propriocep-

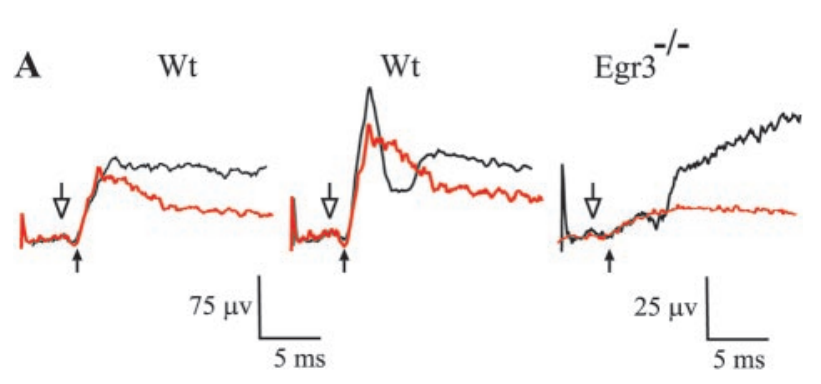

B Quadriceps Obturator Soleus
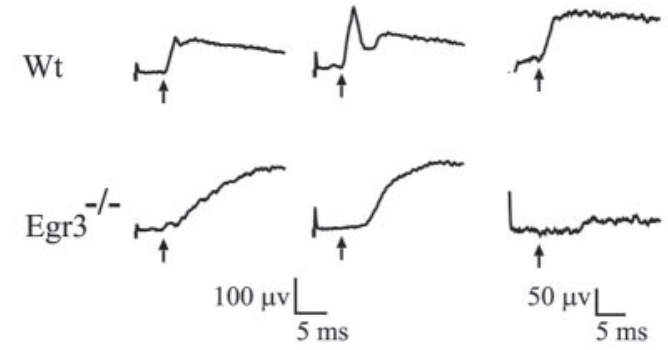

Figure 1. A, Method for measuring monosynaptic components of composite synaptic potentials. All three traces show synaptic input from the obturator nerve recorded extracellularly from the L3 ventral root at P8. The monosynaptic model, shown in red, is scaled vertically to fit the rising phase of the synaptic potential. Terminal field potentials, indicated by open arrows, show the time at which Ia impulses arrive in the ventral horn. See Materials and Methods and Results for more details. Filled arrows in $A$ and $B$ indicate the beginning of the monosynaptic component. $B$, Monosynaptic EPSPs are severely reduced in Egr3 ${ }^{-1-}$ mice. Electrical stimulation of quadriceps, obturator, and soleus muscle nerves at P8 elicits EPSPs in motor axons recorded extracellularly from the ventral root. In normal mice [wild type $(W t)]$, the EPSPs consist of an early $(\sim 5$ msec latency), monosynaptic component from Ia fibers and a later, polysynaptic component from both Ia and Ib fibers. The early monosynaptic component is almost entirely missing in $\mathrm{Egr} 3^{-/-}$mice, although the late polysynaptic component often remains.

tive sensory neurons located in the DRG project peripherally to two types of receptors in the muscles: muscle spindles responsive to stretch (Ia afferents) and Golgi tendon organ (GTO) receptors responsive to tension ( $\mathrm{Ib}$ afferents). Within the spinal cord, these afferents make direct monosynaptic connections with motoneurons (Ia afferents only) and indirect connections via interneurons (Ia and Ib afferents). To determine the cause of the ataxia in mutant mice, we measured the synaptic inputs to motoneurons by recording extracellularly from motor axons in the ventral root while stimulating individual muscle nerves. Normally, the synaptic response consists of a short latency $(\sim 5 \mathrm{msec})$ EPSP corresponding to monosynaptic inputs followed by later, polysynaptic components. The monosynaptic component is often sufficiently large enough to elicit an action potential, as illustrated for the input from the obturator nerve in Figure 1.

In Egr $3^{-/-}$mice, monosynaptic EPSPs are severely reduced in amplitude, although later polysynaptic components often remain (Fig. 1). Monosynaptic components were identified as described in Materials and Methods; in general, they occur 1-2 msec after the terminal field potential (Fig. $1 A$ ). On average, there is a $>10$-fold reduction in the monosynaptic component at P8 (8.1 \pm $9.9 \%$ of control; $p<0.001 ; n=19$ wild type or $+/-; n=35-/-$ ) (summarized in Fig. $7 B$ ). This reduction was seen for all muscle 
Figure 2. GTO (Ib) afferents respond normally in $\mathrm{Egr}^{-1-}$ mice. $A$, A single muscle twitch evokes a burst of Ib spikes recorded from the dorsal root. $B$, Poststimulus time histograms of Ib activity ( $\sim 20$ trials, shown as spikes/sec) show the similar time course of these responses in Egr $3^{-1-}$ and wild-type (Wt) mice. $C, \mathrm{~A}$ burst of Ib activity evoked by a muscle twitch elicits a delayed synaptic response in motoneurons of both normal and mutant mice.
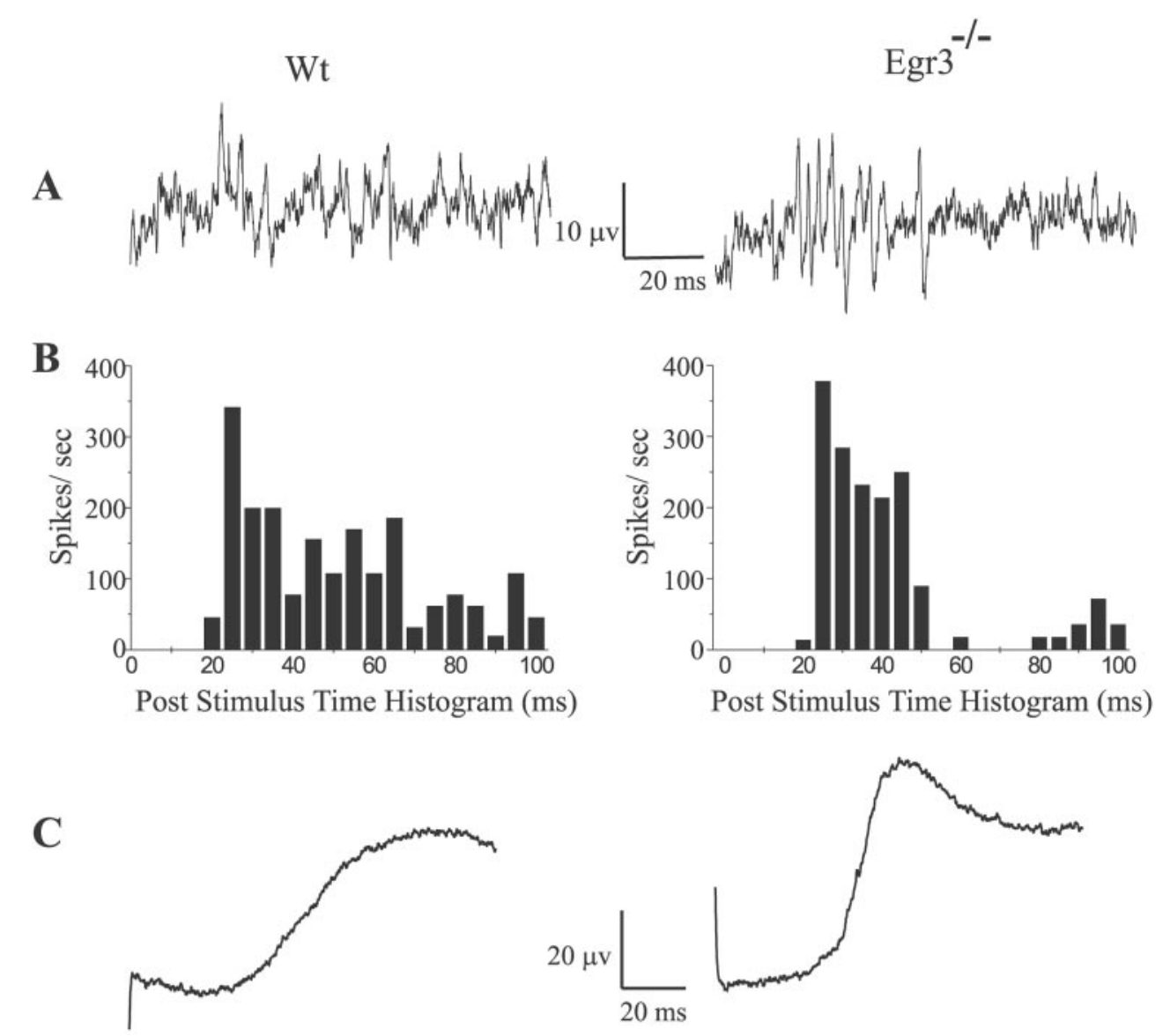

nerves studied at P8, including the quadriceps, obturator, soleus, and medial and lateral gastrocnemius. The loss of monosynaptic input to motoneurons provides a likely explanation for the ataxia. Moreover, because only Ia afferents make monosynaptic connections with motoneurons, these results imply that monosynaptic Ia connections are disrupted, although polysynaptic Ia and Ib inputs might be affected as well. To determine which proprioceptive inputs are lost, we selectively stimulated $\mathrm{Ib}$ afferents.

$\mathrm{Ib}$ afferents were selectively stimulated by eliciting a muscle twitch via electrical stimulation of quadriceps motor axons in the peripheral end of the cut ventral root. The twitch unloads muscle spindles, so there is no activation of stretch-sensitive group Ia or II afferents. However, Ib fibers supplying GTOs, located in series with extrafusal muscle fibers, are activated (Arber et al., 2000). The resulting burst of Ib activity was recorded in the peripheral end of the cut dorsal root. Ib responses are observed in both wild-type and mutant mice (Fig. $2 A$ ), and the poststimulus time histograms of these responses are very similar (Fig. $2 B$ ), suggesting that $\mathrm{Ib}$ afferents respond normally to muscle contractions in mutant mice. Moreover, when dorsal roots are left intact, composite synaptic responses resulting from the burst of Ib impulses are detected in the ventral roots of both wild-type and mutant mice (Fig. 2C). Polysynaptic connections of Ib afferents via interneurons with motoneurons are therefore qualitatively normal in $\mathrm{Egr}^{-1-}$ mice.

\section{la afferents in Egr3 ${ }^{-/-}$mice have normal peripheral and central projections}

The loss of synaptic input from Ia afferents to motoneurons might result from several causes. Although $\operatorname{trkC}^{+}$sensory neurons, which include Ia afferents, are present in normal numbers in the perinatal period (Tourtellotte and Milbrandt, 1998), these afferents might not project peripherally to muscles or centrally to the lateral motor column, where they contact motoneurons. At birth, nerves supplying the soleus and medial gastrocnemius muscles in Egr3 ${ }^{-1-}$ mice contain normal numbers of myelinated axons, implying that Ia afferents, as well as other sensory and motor axons, project normally to muscles (Tourtellotte et al., 2001). In adult mutant mice, however, the number of myelinated axons falls to $75 \%$ of normal. This loss is restricted to group Ia (and II) sensory axons and $\gamma$ motor axons; Ib axons innervating GTOs, which persist in mutant mice, are present in normal numbers (Tourtellotte et al., 2001). To determine whether there is a functional loss of Ia axons in peripheral nerves at P6-P9, we stimulated individual muscle nerves and recorded sensory action potentials in the dorsal root. The amplitudes and latencies of the compound action potentials are normal and sensory axons respond without failure to stimulation frequencies of at least $20 \mathrm{~Hz}$ (data not shown). The latency of the terminal field potential provides a more direct measure of the conduction velocity of Ia axons, because these are the only axons that project into the ventral horn. Measurements of these latencies (examples shown in Fig. $1 A$; see Materials and Methods) showed that Ia axons in mutant mice have normal conduction velocities during the first postnatal week. Therefore, Ia axons in mutant mice conduct action potentials normally.

We traced the central projections of Ia afferents within the spinal cord by applying HRP to dorsal roots, labeling both cutaneous and muscle sensory axons, in P8 mice. Sensory projections 

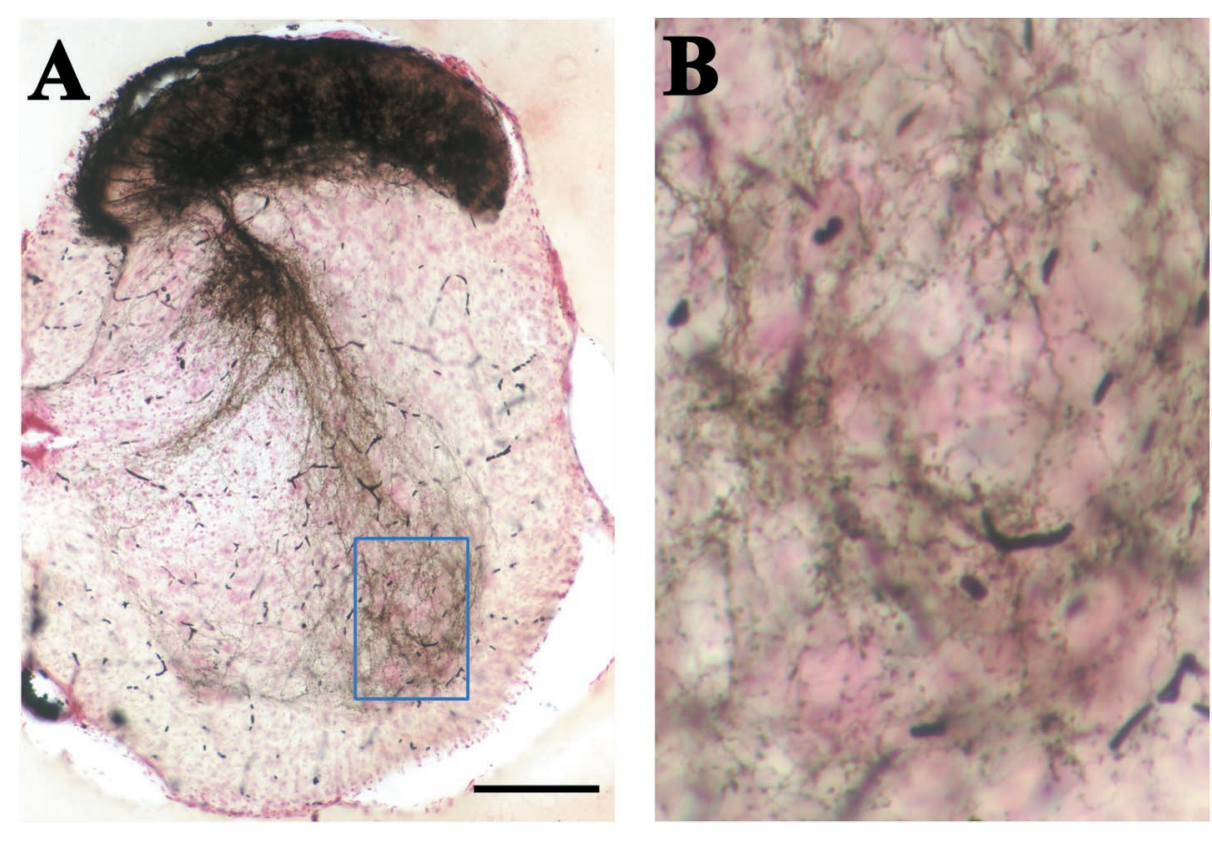

Figure 3. Central projections of sensory neurons appear anatomically normal in Egr3 ${ }^{-/-}$ mice at P8. Sensory axons including Ia afferents were labeled with HRP from the L3 dorsal root. There is a prominent projection, characteristic of Ia collateral axons, ventral to motoneurons in the lateral motor column at $\mathrm{L} 3$ in the vicinity of the quadriceps motor pool $(A)$. Scale bar, 200 $\mu \mathrm{m}$. $B$, At higher magnification of the region outlined in blue (inset in $A$ ), the terminal arborizations of Ia axons contain large numbers of swellings that appear to be synaptic boutons. within the spinal cord appeared to be normal (Fig. 3). In particular, many sensory axons projected ventrally into the lateral motor column and terminated in large numbers of swellings in the vicinity of motoneuronal dendrites, which is the normal termination pattern of Ia afferents. Moreover, Ia impulses were conducted directly into these terminal arborizations. The close proximity between Ia arbors and motoneuronal dendrites results in the presence of a terminal field potential in recordings from motoneurons (Watt et al., 1976; Munson and Sypert, 1979; Arber et al., 2000). These potentials are visible in most of the ventral root recordings from Egr3 mutant mice, just as in normal mice (Fig. 1A). Thus, Ia axons project and conduct impulses to the appropriate regions of the spinal cord despite their inability to form functional monosynaptic connections with motoneurons.

\section{la afferents in Egr3 ${ }^{-/-}$mice respond to muscle stretch}

In Egr3 mutant mice, the number of muscle spindles is normal at birth, but the spindles progressively disassemble and have disappeared in most muscles by adulthood (Tourtellotte et al., 2001). We examined whether this postnatal degeneration of spindles influenced the response properties of Ia axons at P6-P9. Ia axons respond selectively to rapid, small-amplitude vibration of the distal tendon (see Materials and Methods) (Brown et al., 1967; Lichtman and Frank, 1984; Arber et al., 2000). Because the muscle is not contracting, Ib afferents are not activated by these small, passive stretches. Small $(\sim 50 \mu \mathrm{m})$ displacements of the distal tendon of the soleus or rectus femoris (of quadriceps) muscle at low frequency $(1 \mathrm{~Hz})$ elicited similar responses in the dorsal roots of normal and mutant mice (Fig. $4 A$ ). Ia afferents therefore still have stretch-sensitive endings in muscle, consistent with the morphological observations of newborn mutant mice (Tourtellotte et al., 2001). Nevertheless, there is a progressive failure in the Ia responses in mutant versus normal mice with higher-frequency stimulation; at $50 \mathrm{~Hz}$, no Ia response was detected in any of five quadriceps or three soleus preparations from Egr3 ${ }^{-1-}$ mice, whereas all normal preparations respond to this frequency (Arber et al., 2000) (Fig. 4A). Thus, although Ia afferents project to muscles in mutant mice, their innervation of spindles may not be normal. Alternatively, intrinsic defects in
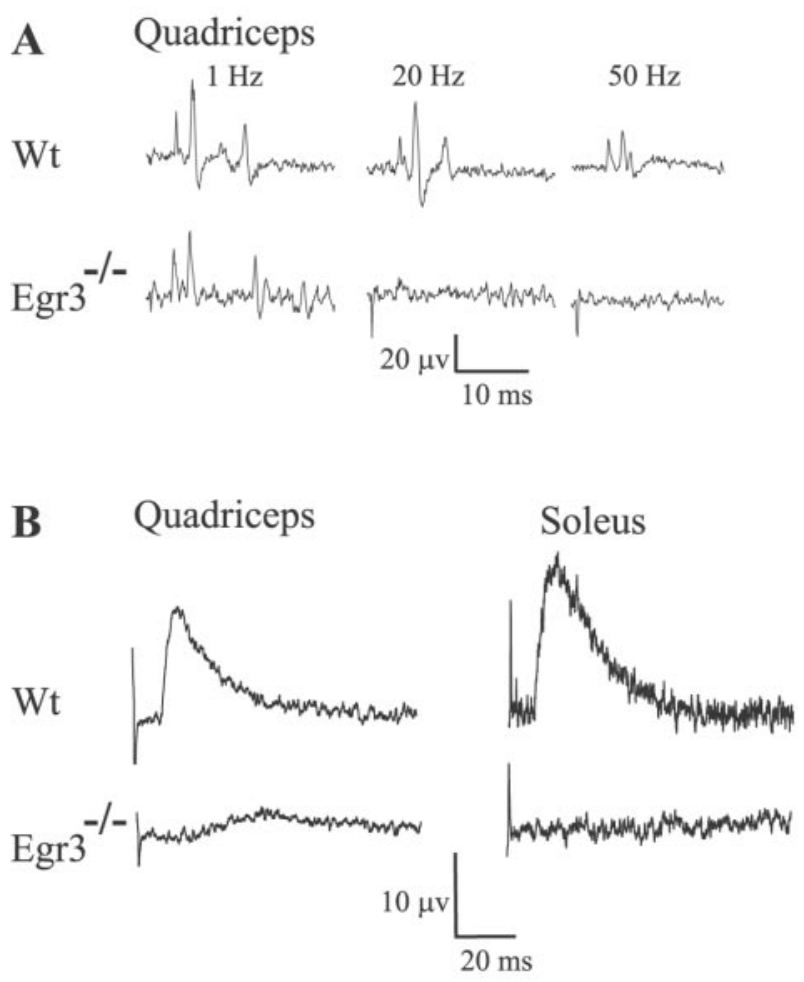

Figure 4. Ia afferents in Egr $3^{-/-}$mice respond to low-frequency muscle stretch but fail to elicit monosynaptic EPSPs. $A$, At a $1 \mathrm{~Hz}$ repetition rate, small-amplitude passive muscle stretches evoke a burst of Ia spikes in both normal and mutant mice. When the stretches are applied at higher rates, however, spindles in mutant muscles fail to respond. $B$, Selective low frequency $(0.5 \mathrm{~Hz})$ stimulation of Ia afferents in quadriceps and soleus muscles elicits a subthreshold monosynaptic EPSP in motoneurons of wild-type (Wt) but not Egr $3^{-1-}$ mice.

Egr3 ${ }^{-/-}$spindles (Tourtellotte et al., 2001) may result in decreased responsiveness.

Selective activation of Ia afferents also made it possible to determine directly whether the Ia monosynaptic input to mo- 

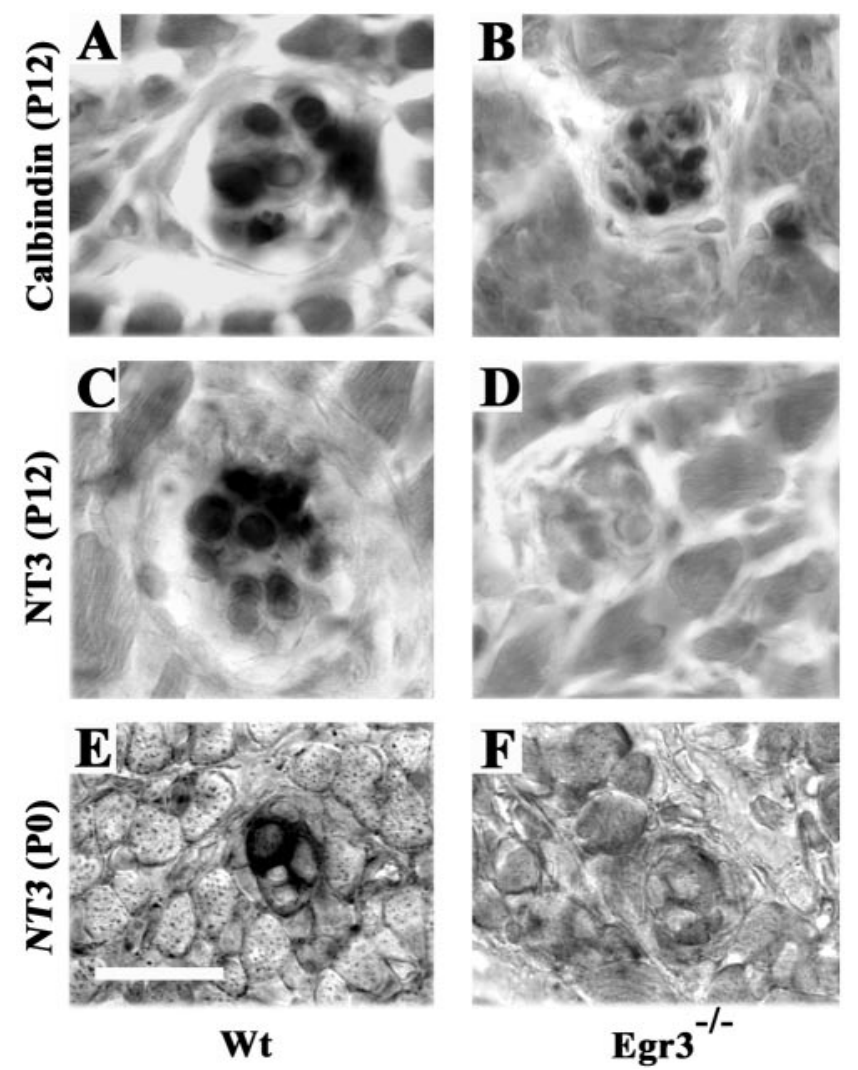

Figure 5. Muscle spindles in Egr $3^{-1-}$ mice do not express NT3. $A, B$, Calbindin staining was used to identify spindles at P12. Spindles in Egr $3^{-/}$ muscles are smaller and reduced in number compared with normal [wildtype $(W t)]$ mice. $C, D$, Mutant spindles at P12 are not immunopositive for NT3, unlike normal spindles. $E, F$, At P0, normal spindles express NT3 mRNA, but mutant spindles do not. Scale bar, $40 \mu \mathrm{m}$.

toneurons is abolished in Egr3 ${ }^{-1-}$ mice. As shown in Figure $4 B$, single quick muscle stretches elicit subthreshold monosynaptic EPSPs in normal mice but not in Egr $3^{-1-}$ mice, confirming that Ia input to motoneurons is largely abolished. Whether polysynaptic connections of Ia afferents are also affected is not clear, because the small number of Ia afferents activated with this technique is insufficient to elicit polysynaptic components even in normal mice.

\section{Muscle spindles in Egr3 ${ }^{-/-}$mice do not express NT3}

Egr3 is expressed in the muscle fibers within spindles but not in sensory or motor neurons. Thus, the loss of synaptic connectivity between sensory and motor neurons is likely to result from a defect in the mutant spindles. One possibility is that muscle spindles in Egr3 mutant mice fail to produce a trophic factor required for normal sensory function. NT3 is a likely candidate, because it is normally produced postnatally in muscle spindles (Copray and Brouwer, 1994) and, when added exogenously in neonates, potentiates the monosynaptic Ia input to motoneurons (Seebach et al., 1999). In Egr3 ${ }^{-1-}$ mice, more than two-thirds of the spindles in quadriceps muscles have disappeared by P12 compared with wild type (17 vs 48 spindles). The remaining spindles are poorly encapsulated and $\sim 50 \%$ smaller than wildtype spindles (Fig. 5A,B). However, unlike spindles in normal mice that are immunopositive for NT3, the spindles that remain in mutant mice are NT3-negative (Fig. 5C,D). Loss of NT3 mRNA expression in spindles was observed even in newborn

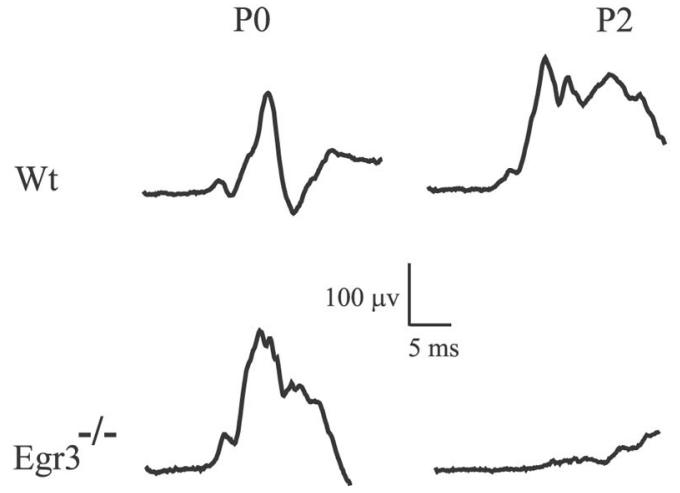

Figure 6. Synaptic connections in $\mathrm{Egr} 3^{-1-}$ mice are functional at birth but are lost by $\mathrm{P} 2$. Synaptic responses in motoneurons at $\mathrm{P} 0$ and $\mathrm{P} 2$ were evoked by electrical stimulation of the quadriceps muscle nerve and recorded in the $\mathrm{L} 3$ ventral root. $W t$, Wild type.

mutant mice, suggesting a spindle-intrinsic defect in NT3 expression (Fig. 5E, $F$ ). Therefore, the absence of NT3 in mutant spindles might be responsible for the synaptic dysfunction seen in these mice.

\section{Synaptic connections in $\mathrm{Egr}^{-/-}$mice are functional at birth but fail by P2}

If NT3 is critical for maintaining functional synaptic transmission between Ia afferents and motoneurons, then these synapses might still be functional at birth, because NT3 is made by extrafusal muscle fibers until this time (Copray and Brouwer, 1994). Consistent with this prediction, electrical stimulation of muscle nerves elicits EPSPs in motoneurons of $\mathrm{Egr}^{-/-}$mice at P0, just as in wild-type mice (Fig. 6). However, by P2, as levels of NT3 in extrafusal muscle fibers fall (Taylor et al., 2001), EPSPs in mutant mice disappear (Fig. 6), although many spindles are still present and Ia afferents are still sensitive to muscle stretch. Thus, the presence of stretch-sensitive spindles in Egr3-null mutant mice is not sufficient to maintain functional synaptic connections. If the lack of NT3 is responsible for this deficit, then application of exogenous NT3 should restore normal transmission.

\section{NT3 rescues functional synaptic connections in $\mathrm{Egr}^{-/-}$mice}

To test directly whether NT3 is required for the maintenance of functional synaptic connections postnatally, we injected NT3 into the right hindlimbs of Egr3 ${ }^{-/-}$mice. For all hindlimb muscle nerves tested, NT3 treatment during the first postnatal week maintained functional sensory-motor connections in mutant mice (Fig. 7A). Surprisingly, sensory-motor synapses were restored both ipsilateral and contralateral to the site of injection (data from both sides are combined in Fig. $7 B$ ). This probably reflects a systemic effect of NT3 attributable to the high dose. No significant difference in synaptic latency was observed in the mutant mice with NT3 treatment, showing that the conduction velocity of Ia afferents is normal (i.e., the axons are not atrophic). Thus, functional rescue of Ia afferents in all nerves we examined appears to be quite complete. Moreover, treatment with NT3 (from $\mathrm{P} 1$ to $\mathrm{P} 7$ ) potentiated synaptic responses $\sim 50 \%$ above normal in both wild-type $(p<0.001)$ and mutant $(p<0.005)$ animals (Fig. $7 B$ ). These data suggest that even in normal mice, synaptic connectivity is limited by the amount of NT3 released from muscle spindles. 
A Quadriceps Obturator Soleus

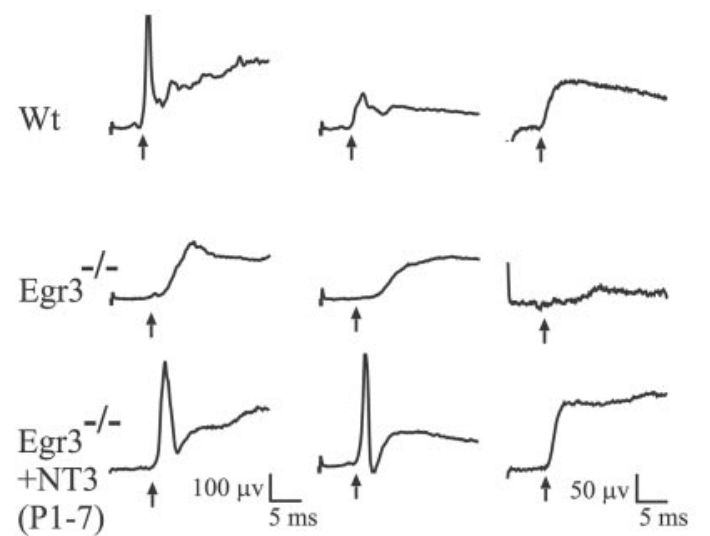

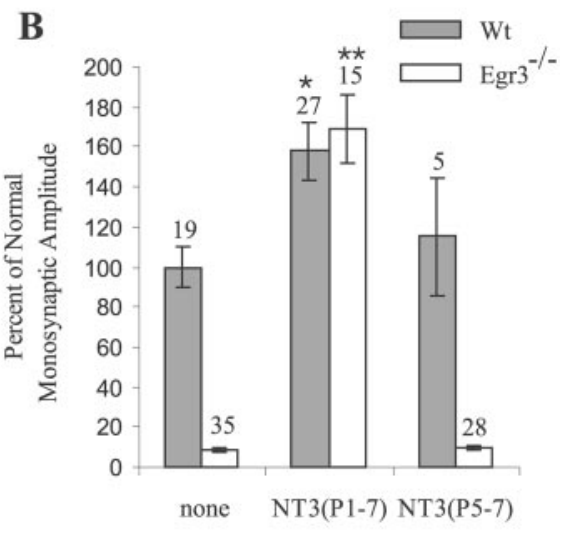

Figure 7. Postnatal NT3 injections restore functional monosynaptic connections in $\mathrm{Egr} 3^{-1-}$ mice. $A$, Intramuscular NT3 injection during the first postnatal week at P1, P3, P5, and P7 [NT3(P1-7)] maintains functional synaptic connections. Representative records of synaptic responses in $\mathrm{Egr}^{-1-}$ mice are shown with or without NT3 injection relative to age-matched wild-type $(W t)$ mice. Arrows indicate the beginning of monosynaptic components. $B$, Analysis of EPSP amplitudes after NT3 injections. Four NT3 injections in the first postnatal week potentiated monosynaptic amplitude in wild-type mice $\left({ }^{*} p<\right.$ $0.001)$ and in mutant mice (**p $<$ $0.005)$. Three daily injections of NT3 between P5 and P7 [NT3(P5-7)] did not potentiate the monosynaptic response in wild-type mice $(p=0.55)$, nor did they restore monosynaptic responses in mutant mice $(p=0.18$ vs mutants with no NT3 treatment). The number above each bar represents the numbers of cases examined.
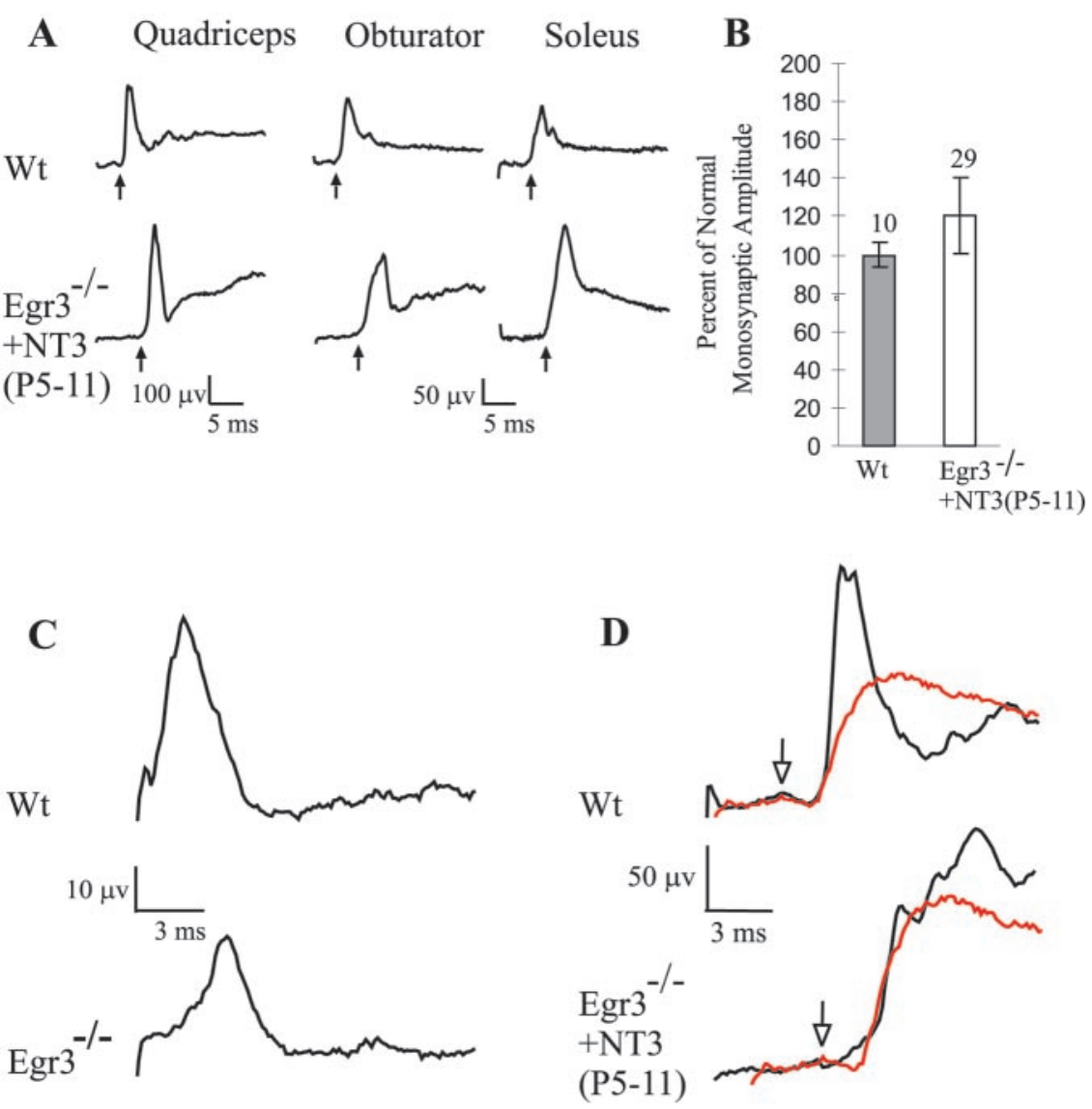

Figure 8. $A, B$, Delayed injection of NT3 into Egr $3^{-1-}$ mice during the second postnatal week at P5, $\mathrm{P} 7, \mathrm{P} 9$, and P11 [NT3(P5-11)] restores synaptic connections. Monosynaptic responses in Egr $3^{-1-}$ mice with delayed NT3 injections are as large as in normal mice but are not potentiated relative to age-matched wild-type $(W t)$ mice $(p=0.54)$. Filled arrows indicate the beginning of monosynaptic components. Gray bars, Wild type; white bars, Egr $3^{-1-}$. C, Delayed NT3 treatment does not fully restore conduction velocities in sensory axons. Traces are dorsal root recordings of quadriceps sensory axons at P12. Some axons in NT3treated mutant mice conduct as rapidly as normal axons (presumably Ib axons), but others have an additional conduction delay of $1-2 \mathrm{msec} . D$, The terminal field potentials of Ia axons are also delayed (open arrows), resulting in an increase in the latency of the monosynaptic response. Traces are ventral root recordings of quadriceps input at P12.
To determine whether NT3 can also restore synaptic connections even after they become nonfunctional, we delayed injection of NT3 for 5 d postnatally, well after monosynaptic connections between Ia afferents and motoneurons are lost. When NT3 treatments are continued for 1 week, from P5 to P11, synaptic connections are restored (Fig. $8 A, B$ ). Delayed treatment with NT3, however, does not prevent Ia atrophy. The peripheral conduction velocity of muscle sensory neurons is reduced, as shown in dorsal root recordings in Figure $8 C$. The additional conduction time results in an increase of $2-3 \mathrm{msec}$ in the latency of the terminal field potentials (Fig. 8D) and of the monosynaptic responses (Fig.
$8 A, D)$. Therefore, even after synaptic transmission has failed, NT3 can restore functional synapses. However, when mutant mice are treated with NT3 for only $3 \mathrm{~d}$, between P5 and P7, synaptic connections are not restored when tested on P8 (Fig. 7B). The effect of NT3 on transmission at these later times thus requires exposure over several days.

\section{DISCUSSION}

Several lines of evidence suggest that postnatal production of NT3 by muscle spindles is required for trophic support of Ia afferents, although this hypothesis has never been directly tested 
in vivo. In contrast to its expression by extrafusal fibers prenatally, NT3 expression becomes selectively restricted to the fibers of muscle spindles after birth (Copray and Brouwer, 1994). NT3 receptors (trkC) are expressed in proprioceptive muscle afferents during embryonic development and throughout adulthood (McMahon et al., 1994). Axotomy of peripheral nerves in adult cats causes a reduction in the strength of monosynaptic connections between Ia afferents and motoneurons (Goldring et al., 1980), and administration of NT3 to the proximal nerve stump prevents this loss of synaptic connectivity (Munson et al., 1997). However, it was not known whether the effect of spindle-derived NT3 on maintaining Ia afferent central synapses is part of its normal physiological function during development or an effect triggered by axotomy, in which the sensitivity of sensory neurons to neurotrophins may be altered (Curtis et al., 1998; Tonra et al., 1998). Using $\mathrm{Egr}^{-1-}$ mice that have a progressive degeneration of spindles after birth, we provide direct evidence for a normal postnatal function of spindle-derived NT3.

In Egr $3^{-1-}$ mice, central synapses between Ia afferents and motoneurons fail as early as P2. Because loss of functional transmission precedes loss of morphologically distinct muscle spindles, the presence of spindles is not sufficient to support central synapses by Ia afferents. The failure of synaptic transmission in $\mathrm{Egr}^{-1-}$ mice coincides with the absence of NT3 production from muscle spindles. Moreover, exogenous NT3 treatment in mutant mice restores central synapses. Therefore, NT3 derived from muscle spindles is necessary to maintain central synapses between Ia sensory and motor neurons in early postnatal development.

Our results also show that NT3 is not required postnatally for the continued survival of Ia or Ib afferents. Null mutations for NT3 or trkC result in the death of virtually all proprioceptive neurons, but it was not known whether the requirement for this signaling pathway extended into the neonatal period. Many sensory neurons die if peripheral nerves are transected in neonates (Zelena, 1994), but it is not known to what extent the loss of a peripheral source of NT3 is responsible for this effect. Despite the absence of NT3 in Egr $3^{-/-}$spindles, Ia afferents continue to survive for at least several weeks (Tourtellotte and Milbrandt, 1998) and, as demonstrated here, continue to respond to muscle stretch, albeit with a reduced sensitivity. It will be interesting to see whether spindles provide other factors that are required postnatally for Ia afferent survival or synaptic function.

Recently, Arvanov et al. (2000) reported that direct exposure of isolated spinal cords to NT3 potentiates the monosynaptic sensory-motor connections within $20 \mathrm{~min}$ in neonatal rats. In the present experiments, a direct effect of NT3 on central synapses also might be responsible for the maintenance of synaptic connections in mutant mice when NT3 is administered in the immediate postnatal period. The blood-brain barrier is not well developed at this time, so NT3 injected into the limb may act directly on central synapses. The delayed effects of NT3 injected from P5 to P11 reported here are likely to be mediated differently, however. These effects are not acute, because three daily injections of NT3 at the end of the first postnatal week are insufficient to restore synaptic transmission. One possible explanation for the slower effect we observe is that the restoration of the fine terminal arborizations of Ia collaterals requires prolonged exposure to NT3. NT3 is known to promote DRG neuron arborization in vitro (Lentz et al., 1999) and to promote axonal branching in a layer-specific manner during the development of the mammalian cerebral cortex (Castellani and Bolz, 1999).
Alternatively, synaptic contacts in $\operatorname{Egr} 3^{-/-}$mice might be morphologically normal, but some aspect of neurosecretion could be disturbed. The number of synaptic vesicles or their exocytotic/ endocytotic cycle might be altered by NT3-trkC signaling. In trkC-null mutant mice, the presynaptic proteins responsible for synaptic vesicle fusion, including synapsin 1, vesicle-soluble $N$-ethylmaleimide-sensitive factor attachment protein receptor (SNARE), and SNARE on target membrane, are dramatically downregulated in the hippocampus (Blasi et al., 1993; Sudhof, 1995; Geppert et al., 1997; Hay and Scheller, 1997). Conversely, at the neuromuscular junction, NT3 produced from embryonic muscle can promote maturation of developing neuromuscular synapses by increasing the levels of the synaptic vesicle proteins synapsin I and synaptophysin and thus increasing neurotransmitter release from motoneurons (Lohof et al., 1993; Wang et al., 1995; Liou and Fu, 1997; Xie et al., 1997). Moreover, NT3 production by embryonic muscle is itself activity dependent, providing a positive feedback mechanism to establish the neuromuscular junction (Xie et al., 1997). If NT3 release from intrafusal muscle fibers were similarly dependent on spindle activity or on mechanical stretching of the spindle, then the strength of the monosynaptic stretch reflex could be modulated by stretchevoked activity throughout adult life.

\section{REFERENCES}

Arber S, Ladle DR, Lin JH, Frank E, Jessell TM (2000) ETS gene Er81 controls the formation of functional connections between group Ia sensory afferents and motor neurons. Cell 101:485-498.

Arvanov VL, Seebach BS, Mendell LM (2000) NT-3 evokes an LTP-like facilitation of AMPA/kainate receptor-mediated synaptic transmission in the neonatal rat spinal cord. J Neurophysiol 84:752-758.

Blasi J, Chapman ER, Link E, Binz T, Yamasaki S, De Camilli P, Sudhof TC, Niemann H, Jahn R (1993) Botulinum neurotoxin A selectively cleaves the synaptic protein SNAP-25. Nature 365:160-163.

Brown MC, Engberg I, Matthews PB (1967) The relative sensitivity to vibration of muscle receptors of the cat. J Physiol (Lond) 192:773-800.

Carr VM, Simpson Jr SB (1978) Proliferative and degenerative events in the early development of chick dorsal root ganglia. I. Normal development. J Comp Neurol 182:727-739.

Castellani V, Bolz J (1999) Opposing roles for neurotrophin-3 in targeting and collateral formation of distinct sets of developing cortical neurons. Development 126:3335-3345.

Copray JC, Brouwer N (1994) Selective expression of neurotrophin-3 messenger RNA in muscle spindles of the rat. Neuroscience 63:1125-1135.

Curtis R, Tonra JR, Stark JL, Adryan KM, Park JS, Cliffer KD, Lindsay RM, DiStefano PS (1998) Neuronal injury increases retrograde axonal transport of the neurotrophins to spinal sensory neurons and motor neurons via multiple receptor mechanisms. Mol Cell Neurosci 12:105-118

Ernfors P, Lee KF, Kucera J, Jaenisch R (1994) Lack of neurotrophin-3 leads to deficiencies in the peripheral nervous system and loss of limb proprioceptive afferents. Cell 77:503-512.

Farinas I, Jones KR, Backus C, Wang XY, Reichardt LF (1994) Severe sensory and sympathetic deficits in mice lacking neurotrophin-3. Nature 369:658-661.

Geppert M, Goda Y, Stevens CF, Sudhof TC (1997) The small GTPbinding protein Rab3A regulates a late step in synaptic vesicle fusion. Nature 387:810-814.

Goldring JM, Kuno M, Nunez R, Snider WD (1980) Reaction of synapses on motoneurones to section and restoration of peripheral sensory connexions in the cat. J Physiol (Lond) 309:185-198.

Hamburger V, Brunso-Bechtold JK, Yip JW (1981) Neuronal death in the spinal ganglia of the chick embryo and its reduction by nerve growth factor. J Neurosci 1:60-71.

Hay JC, Scheller RH (1997) SNAREs and NSF in targeted membrane fusion. Curr Opin Cell Biol 9:505-512.

Jansen JKS, Rudjord T (1964) On the silent period and Golgi tendon organs of the soleus muscle of the cat. Acta Physiol Scand 62:364-379.

Kucera J, Walro JM (1987) Postnatal maturation of spindles in deafferented rat soleus muscles. Anat Embryol 176:449-461.

Kucera J, Walro JM (1988) The effect of neonatal deafferentation or deefferentation on the immunocytochemistry of muscle spindles in the rat. Neurosci Lett 95:88-92.

Lentz SI, Knudson CM, Korsmeyer SJ, Snider WD (1999) Neurotro- 
phins support the development of diverse sensory axon morphologies. J Neurosci 19:1038-1048.

Lichtman JW, Frank E (1984) Physiological evidence for specificity of synaptic connections between individual sensory and motor neurons in the brachial spinal cord of the bullfrog. J Neurosci 4:1745-1753.

Liou JC, Fu WM (1997) Regulation of quantal secretion from developing motoneurons by postsynaptic activity-dependent release of NT-3. J Neurosci 17:2459-2468.

Lohof AM, Ip NY, Poo MM (1993) Potentiation of developing neuromuscular synapses by the neurotrophins NT-3 and BDNF. Nature 363:350-353

McMahon SB, Armanini MP, Ling LH, Phillips HS (1994) Expression and coexpression of Trk receptors in subpopulations of adult primary sensory neurons projecting to identified peripheral targets. Neuron 12:1161-1171.

Mears SC, Frank E (1997) Formation of specific monosynaptic connections between muscle spindle afferents and motoneurons in the mouse. J Neurosci 17:3128-3135.

Mendell LM, Johnson RD, Munson JB (1999) Neurotrophin modulation of the monosynaptic reflex after peripheral nerve transection. J Neurosci 19:3162-3170.

Munson JB, Sypert GW (1979) Properties of single central Ia afferent fibres projecting to motoneurones. J Physiol (Lond) 296:315-327.

Munson JB, Johnson RD, Mendell LM (1997) NT-3 increases amplitude of EPSPs produced by axotomized group Ia afferents. J Neurophysiol 77:2209-2212.

Oakley RA, Garner AS, Large TH, Frank E (1995) Muscle sensory neurons require neurotrophin-3 from peripheral tissue during the period of normal cell death. Development 121:1341-1350.

Oakley RA, Lefcort FB, Clary DO, Reichardt LF, Prevette D, Oppenheim RW, Frank E (1997) Neurotrophin-3 promotes the differentiation of muscle spindle afferents in the absence of peripheral targets. J Neurosci 17:4262-4274.

Sah DW, Frank E (1984) Regeneration of sensory-motor synapses in the spinal cord of the bullfrog. J Neurosci 4:2784-2791.

Seebach BS, Arvanov V, Mendell LM (1999) Effects of BDNF and NT-3 on development of Ia/motoneuron functional connectivity in neonatal rats. J Neurophysiol 81:2398-2405.
Sudhof TC (1995) The synaptic vesicle cycle: a cascade of proteinprotein interactions. Nature 375:645-653.

Taylor MD, Vancura R, Patterson CL, Williams JM, Riekhof JT, Wright DE (2001) Postnatal regulation of limb proprioception by musclederived neurotrophin-3. J Comp Neurol 432:244-258.

Tessarollo L, Vogel KS, Palko ME, Reid SW, Parada LF (1994) Targeted mutation in the neurotrophin-3 gene results in loss of muscle sensory neurons. Proc Natl Acad Sci USA 91:11844-11848.

Tojo H, Kaisho Y, Nakata M, Matsuoka K, Kitagawa M, Abe T, Takami K, Yamamoto M, Shino A, Igarashi K, Aizawa S, Shio O (1995) Targeted disruption of the neurotrophin-3 gene with lacZ induces loss of trkC-positive neurons in sensory ganglia but not in spinal cords. Brain Res 669:163-175.

Tonra JR, Curtis R, Wong V, Cliffer KD, Park JS, Timmes A, Nguyen T, Lindsay RM, Acheson A, DiStefano PS (1998) Axotomy upregulates the anterograde transport and expression of brain-derived neurotrophic factor by sensory neurons. J Neurosci 18:4374-4383.

Tourtellotte WG, Milbrandt J (1998) Sensory ataxia and muscle spindle agenesis in mice lacking the transcription factor Egr3. Nat Genet 20:87-91.

Tourtellotte WG, Keller-Peck C, Milbrandt J, Kucera J (2001) The transcription factor Egr3 modulates sensory axon-myotube interactions during muscle spindle morphogenesis. Dev Biol 232:388-399.

Wang T, Xie K, Lu B (1995) Neurotrophins promote maturation of developing neuromuscular synapses. J Neurosci 15:4796-4805.

Watt DG, Stauffer EK, Taylor A, Reinking RM, Stuart DG (1976) Analysis of muscle receptor connections by spike-triggered averaging. I. Spindle primary and tendon organ afferents. J Neurophysiol 39:1375-1392.

Wright DE, Zhou L, Kucera J, Snider WD (1997) Introduction of a neurotrophin-3 transgene into muscle selectively rescues proprioceptive neurons in mice lacking endogenous neurotrophin-3. Neuron 19:503-517.

Xie K, Wang T, Olafsson P, Mizuno K, Lu B (1997) Activity-dependent expression of NT-3 in muscle cells in culture: implications in the development of neuromuscular junctions. J Neurosci 17:2947-2958.

Zelena J (1994) Nerves and mechanoreceptors: the role of innervation in the development and maintenance of mammalian mechanoreceptors. London: Chapman Hall. 